\title{
CONTRIBUTION OF ENERGY EXPENDITURE DURING ACUTE SESSION OF HIGH INTENSITY INTERVAL TRAINING AND CONTINUOUS AEROBIC TRAINING
}

\author{
Alexandre Etechebere (IC)*, Alex Castro (PG), Marcel Lopes dos Santos (IC), Claudia R. Cavaglieri (CO); Mara \\ PatríciaT. Chacon-Mikahil (PQ)
}

\begin{abstract}
High Intensity Interval Training (HIIT) has been pointed as an efficient method for cardiorespiratory enhancement in comparison with traditional continuous aerobic training (CAT). Such difference is believed to be explained by assessing the energy expenditure of training sessions. Hence, in order to test this hypothesis, it was realized an acute intervention in recreationally active young men during three experimental conditions with a cross-over design. Energy expenditure results indicate that it is highly correlated with exercise volume, and even though energetic systems contribution during HIIT and CAT sessions were different, total caloric expenditure were similar between exercises sessions.
\end{abstract}

\section{Key words:}

Aerobic training, energy expenditure, Interval Training

\section{Introduction}

Continuous aerobic training (CAT) is a traditional well established method of cardiorespiratory enhancement ${ }^{1}$. Nevertheless, high intensity interval training (HIIT) has shown more efficient results for cardiorespiratory enhancement in comparison with $\mathrm{CAT}^{2}$. HIIT is characterized by high intensity exercise stimulus interspersed with rest or low intensity active rest intervals ${ }^{3}$. Considering the differences between these two types of training, it is supposed that different metabolic pathways and energy production mechanisms are utilized. Thus, we hypothesized that energy production derived from anaerobic pathway during HIIT is greater than during CAT, which would result in an increase of session energy expenditure. Those results would help to understand the mechanisms by which HIIT can be more efficient for cardiorespiratory enhancement based on a contribution of energetic systems approach.

\section{Results and Discussion}

Eleven young men (18-30 years old) were submitted to two acute experimental sessions: CAT $(40 \mathrm{~min}$ at $70 \%$ of Heart Rate Reserve (HRR)) and HIIT (40 min with 5 bouts of $4 \mathrm{~min}$ at $90 \%$ of HRR intercalated with $3 \mathrm{~min}$ at $50 \%$ of $\mathrm{HRR}$ ). The contribution of the energy systems of training methods was calculated by GEDAE-LAB software ${ }^{4}$. Data were analyzed by a Mixed Model of repeated measures, having experimental sessions and energy systems as fixed factors and subjects as a random factor when It was appropriated. The measure of the effect size was calculated by cohen's d coefficient. Significance level was $5 \%(\mathrm{P}<0.05)$. There was a greater relative energy expenditure of oxidative system during CAT compared to HIIT $(P=0.002)$ and higher relative energy expenditure of lactic system in the HIIT compared to CAT $(P=0.001)$. It also was observed in both HIIT and CAT a greater oxidative energy expenditure in comparison to alactic and lactic energy expenditure $(\mathrm{P}<$ 0.001 for both) and a lower and higher alactic energy expenditure for HIIT $(P=0.007)$ and CAT $(P=0.004)$, respectively, in comparison to lactic energy expenditure. Total energy expenditure was similar between CAT and HIIT sessions $(P=0.166)$.

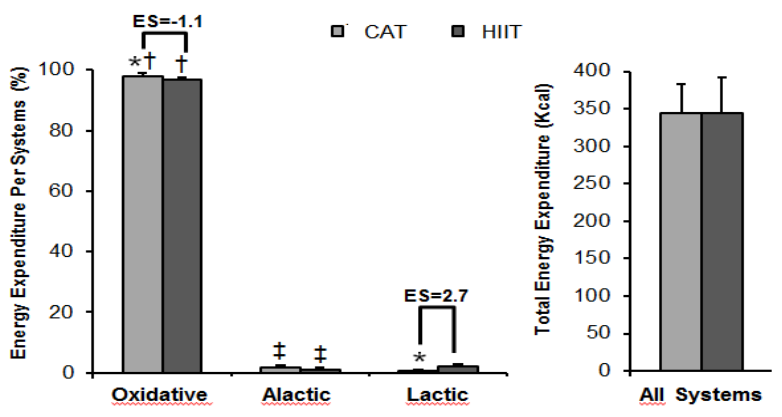

Figure 1: Energy Expenditure. * $\mathrm{P}<0.05$ vs. HIIT. ${ }^{\dagger}$ $\mathrm{P}<0.05$ vs. Alactic and Lactic CAT. $\ddagger \mathrm{P}<0.05$ vs. Lactic. ES = Effect Size (Cohen's d).

\section{Conclusions}

We concluded that, total energy expenditure between sessions was similar, with greater relative oxidative energy expenditure for the CAT and higher relative lactic energy expenditure for the HIIT. This indicates that greater cardiorespiratory adaptations commonly observed after HIIT in comparison to CAT cannot be attributed to greater energy expenditure during HIIT sessions, but likely due to different relative contributions of the energetic systems between CAT and HIIT. Other physiological variables, such as different metabolites expression or oxygen consumption after exercise are suggested to better explain the differences at promoted adaptations by both methods.

\section{Acknowledgement}

\section{FAEPEX, FISEX and LABFEF-FEF-UNICAMP.}

1 ACSM's position stand. Quantity and quality of exercise for developing and maintaining cardiorespiratory, musculoskeletal, and neuromotor fitness in apparently healthy adults: guidance for prescribing exercise. Med Sci Sports Exerc, v. 43, n. 7, p. 1334-1359, 2011.

2 NYBO L.; et al. High-Intensity Training versus Traditional Exercise Interventions for Promoting Health. Med Sci Sports Exerc, v. 42, n. 10, p. 1951-1958, 2010.

3 CIOLAC, E. G. High-intensity interval training and hypertension: maximizing the benefits of exercise? Amer J Cardiovasc Disease, v. 2, n. 2, p. 102-110, 2012.

4 BERTUZZI, R; et al. GEDAE-LaB: A Free Software to Calculate the Energy System Contributions during Exercise. PLoS One, v. 11, n. 1, p. 1-13, 2016.

DOI: 10.19146/pibic-2017-78751 